\title{
Avoidable stillbirths and neonatal deaths in rural Tanzania
}

\author{
Sven G. Hinderaker ${ }^{a, b}, *$, Bjørg E. Olsen ${ }^{a, b}$, Per B. Bergsjø ${ }^{c}$, Peter Gasheka ${ }^{\mathbf{b}}$, \\ Rolv T. Lie ${ }^{d}$, Jakob Havnen ${ }^{\mathrm{e}}$, Gunnar Kvåle ${ }^{\mathrm{a}}$
}

Objective To determine the causes of stillbirths and neonatal deaths in the community in rural Tanzania and to evaluate whether the deaths were avoidable under the prevailing circumstances.

Design Review of stillbirths and neonatal deaths.

Setting Rural northern Tanzania, Mbulu and Hanang districts.

Sample One hundred and nineteen stillbirth and neonatal deaths identified in a prospective cohort of antenatal attendees and 21 stillbirths and neonatal deaths identified retrospectively in a household survey in seven rural communities.

Methods Verbal autopsy was done to reach a diagnosis, in many cases supplemented with information from antenatal records and hospital records. The avoidability of deaths under the prevailing circumstances was assessed for each case. An account of risk factors detectable at antenatal clinic was done and compared with the woman's recall of the risk assessment and recall of being referred.

Main outcome measures Avoidability of stillbirths and neonatal deaths.

Results There were 60 stillbirths, 49 early neonatal deaths and 27 late neonatal deaths. Infection-related deaths were most common $(n=53)$, followed by asphyxia-related deaths $(n=32)$ and immaturity-related deaths $(n=20)$. Malaria was the most common infectious agent observed ( 21 children and 20 mothers). Twentyone deaths $(15 \%)$ were probably avoidable and $13(10 \%)$ were possibly avoidable. A patient-oriented avoidable factor was identified in $17(51 \%)$ and a provider-oriented avoidable factor was identified in 22 cases (65\%). Twenty-six of the 34 avoidable deaths had risk factors, but only two of the women were aware of it and only one recalled being referred to a hospital for the risk factor. There were eight deaths among the 133 mothers who experienced a perinatal death.

Conclusions Our data indicate that prevention and adequate treatment of infections and asphyxia in the newborn should have high priority in low-income settings. The relatively low proportion of avoidable stillbirths and neonatal deaths may be partly due to accessible emergency obstetric care in the area. Future efforts should emphasise improving the communication between midwife and women at the antenatal clinics, preparing the women - and their families - for the delivery and to be ready for complications.

\section{INTRODUCTION}

According to the World Health Report 2001, perinatal conditions account for more than $4 \%$ (2.4 million) of the deaths in the world, most of them in developing countries ${ }^{1}$. Population-based studies in various regions of Tanzania

${ }^{a}$ Centre for International Health, University of Bergen, Norway

${ }^{\mathrm{b}}$ Haydom Lutheran Hospital, Mbulu, Tanzania

${ }^{\mathrm{c} D e p a r t m e n t}$ of Obstetrics and Gynaecology, Haukeland Hospital, Bergen, Norway

${ }^{\mathrm{d}}$ Section for Medical Statistics, University of Bergen, Norway

${ }^{\mathrm{e}}$ Paediatric Department, Rogaland Central Hospital, Stavanger, Norway

* Correspondence: Dr S. G. Hinderaker, Centre for International Health, Armauer Hansen's Building, University of Bergen, N-5021 Bergen, Norway. have shown perinatal mortality rates of $125 / 1000^{2}, 82 /$ $1000^{3}, 68 / 1000^{4}$ and $58 / 1000$ births $^{5}$. Some perinatal and neonatal conditions are almost invariably fatal, whereas others are more easily managed. Therefore, an assessment of avoidability of deaths may help identify the areas most likely to succeed in preventing these deaths and may also give an indication of the performance of the health system and the health-seeking behaviour in the area. It has been suggested that the reduction of perinatal mortality rate in high-income countries was due to improved socioeconomic and environmental conditions ${ }^{6,7}$. Studies on the perinatal mortality in the 19th century suggest that the introduction of midwifery-assisted deliveries was instrumental in reducing the perinatal mortality rate $^{8}$, but more so for reducing maternal mortality ${ }^{9}$. In addition, simple improvements of hospital routines ${ }^{10}$ and perinatal audits ${ }^{11}$ have been shown to reduce perinatal mortality.

A relatively low perinatal mortality rate was observed in the rural districts of Hanang and Mbulu of northern Tanzania (unpublished observations). The objective of the present study was to determine the underlying causes of 
stillbirths and neonatal deaths in the community and to evaluate whether the deaths were avoidable under the prevailing circumstances.

\section{METHODS}

We defined a stillbirth as the birth of a dead fetus of at least 28 weeks of gestation. Early neonatal deaths were deaths of live born babies during the first seven completed days after birth, and late neonatal deaths occurred after 7 completed days and before 28 completed days. Perinatal mortality includes stillbirths and early neonatal deaths, and the rates are given per 1000 births. Neonatal mortality includes both early and late neonatal deaths, and the rates are given per 1000 live births.

The study was conducted within two administrative divisions, Dongobesh in Mbulu district and Basotu in Hanang district, covering 42 villages in the northern highlands of Tanzania. The average altitude is $1700 \mathrm{~m}$ (range 1300-2100) above sea level. Most people in this rural area are peasants with small fields and livestock. The health services are provided by government institutions as well as non-governmental organisations. Delivery facilities are offered at around 10 health centres or dispensaries in the area, but during the study period, caesarean sections were done only at the Haydom Lutheran Hospital. Although antenatal coverage is high in Tanzania, $47 \%$ of the women deliver their babies at home and $66 \%$ deliver without a trained assistant, according to a report from the World Health Organization ${ }^{12}$. In our study area, information from obstetric histories of antenatal clinic attendees indicated that around $70 \%$ of them had their first delivery at a health care facility, whereas around $40 \%$ of subsequent babies were delivered at health care facilities (unpublished observations). The pregnant woman's mother-in-law, who was usually untrained, assisted most of the home deliveries. The maternal mortality ratio, which was studied at the same time, was found to be between 300 and 400 per 100,000 live births and was reported in a separate paper ${ }^{13}$. Cerebral malaria was commonly observed at the local hospital; it was the leading cause of maternal deaths, whereas eclampsia was uncommon. The prevalence of HIV infection among pregnant women was $0.3 \%^{14}$. The nutrition among the pregnant Iraqw and Datoga women was fairly good, and there was no tradition of starving pregnant women like the Masai sometimes do.

To reach diagnoses for causes of deaths of stillborn and neonates, we collected information on children born to women in two populations: antenatal clinic attendees and participants of a household survey in the same area.

A cohort of 3618 antenatal clinic attendees were included in a prospective study, and we managed to trace the outcome of pregnancy for 3512 of them (97.1\%). Most of the women were followed up at the maternal and child health clinics when they brought their newborn babies for vaccination. The women who did not return were traced to their home villages and asked about the outcome of the pregnancy and time of delivery. We registered 119 stillbirths and neonatal deaths in this group.

Independently of the former cohort, perinatal and neonatal deaths were also reported retrospectively from a household survey conducted in seven selected subvillages in the study area. Among the 1282 eligible households, representatives of 1259 were interviewed, 12 were missing, 6 refused and a further 5 refused after the interview had started. Every household was asked about births and deaths during the 12 months prior to the interview. There were 370 children born during the period, and 21 of them resulted in stillbirths or neonatal deaths. One of them was also covered by the above-mentioned antenatal cohort.

Of the total 139 deaths, 75 died at a health facility, and hospital records were available on 58 of these and on 6 cases who had been admitted but died at home or roadside. In 134 cases, we were able to interview the mother or the closest relative about symptoms, signs and circumstances of death (verbal autopsy) to reach a tentative diagnosis. The causes of deaths were based on 134 verbal autopsies, 119 antenatal clinic screening records, 64 history sheets and delivery records at Haydom Lutheran Hospital and 21 household survey interviews.

The verbal autopsy was initially derived from Smith and Morrow $^{15}$, translated into Swahili and tested in the field. The interview consisted of an initial open-ended interview, followed by a questionnaire with closed-ended questions. The interview was done by a medical doctor (authors SGH or BEO) fluent in Swahili and well acquainted with the local cultures and a local field assistant fluent in the other local languages. An experienced obstetrician (author PB) and two medical doctors with local clinical experience (authors SGH and BEO) independently determined the preliminary diagnoses based on the existing information. We kept the diagnoses where at least two doctors agreed; where all three disagreed, we discussed the case and reached consensus. In many cases, we assigned two or more diagnoses; two were found in 60, three in 40 and four diagnoses in 13 cases. The diagnoses were reviewed and commented on by an experienced paediatrician (author JH), which led to change in one underlying cause of death and modifications on 10 of the contributing diagnoses.

The causes of death were grouped into a functional classification developed by Wigglesworth et al. ${ }^{16}$ and modified by the International Collaborative Effort on Birth Weight, Plurality, Perinatal and Infant Mortality ${ }^{17}$. The underlying causes of death were grouped into seven functional categories. Each group has common features requiring intervention at a specific time. (1) 'Congenital anomalies' include both structural and biochemical abnormalities. (2) The 'asphyxia' group comprises conditions arising around delivery, with strategies for prevention being concentrated to that time. (3) The 'immaturity related group' comprises conditions associated with preterm birth 
and small babies. (4) The group comprised by 'infections' includes all kinds of infections regardless of causative agent and localisation. (5) 'Sudden death' includes all cases of sudden death of unknown cause. (6) 'External causes' comprise accidents, poisoning and specific deficiencies. (7) 'Other specific conditions' is a heterogeneous group, including neoplasms and isoimmunisation. (8) We also had an additional 'other and unclassifiable' group, where we included unexplained intrauterine fetal deaths.

According to the information given, we (authors SGH and BEO) also assessed whether the deaths were 'avoidable'. The term referred only to the avoidability under the prevailing circumstances (i.e. given the existing disease patterns, health facilities, infrastructure, resources and routines), hence referring to the immediate actions taken or omitted by the persons involved. Therefore, an 'unavoidable' death may have been caused by both 'treatable' and 'untreatable' conditions. Deaths were registered as possibly 'avoidable' if there was a fair or good chance of survival if the danger signs had been recognised and correct steps had been taken at the appropriate time. The death was regarded 'unavoidable' if there was little or no chance that the baby would have survived, even if the correct decisions had been made in due time. The factors that were suspected to be responsible for the avoidable deaths, were categorised into patient-oriented and health service provider-oriented, the latter including antenatal clinics, health centres, dispensaries and hospitals.

For data entry, we used EpiInfo version 5 and $6^{18}$. For analysis of the data, we used SPSS version 9.0.0 ${ }^{19}$. Differences in proportions were analysed by $\chi^{2}$ tests. The significance level was 0.05 and two-sided.

The research protocol was approved by the National Committee for Research Ethics in Medicine in Norway and by the Commission for Science and Technology in Tanzania. Prior to the field study, the Regional Development Officer, the District Commissioners and the leaders of wards and villages had given consent. The local people had been informed about the study through gatherings in the villages. Individual oral consent was also obtained from each participant.

\section{RESULTS}

Among the 139 registered deaths, there were 60 stillbirths, 49 early neonatal deaths, 27 late neonatal deaths and 3 cases with no information on age at time of death. Among the 76 neonatal deaths, 27 (36\%) died within the first day. Table 1 shows the frequency of the various categories of underlying cause of death for 136 cases. Infection-related deaths dominated, particularly among the late neonatal deaths, followed by asphyxia-related deaths. Among the 53 infection-related deaths, 20 babies had malaria, 11 had pneumonia and 5 had septicaemia. There were 21 cases of maternal malaria and 10 cases of suspected other maternal infections. Among the 32 asphyxia-related deaths, 11 had compression or prolapse of the umbilical cord, 6 babies had signs of aspiration, 5 were difficult breech deliveries and 5 were victims of antepartum haemorrhage. There were 128 singletons, 4 pairs of twins and a group of triplets among the 139 dead babies. Eight of the 133 delivering women died. The three direct maternal deaths resulted in one baby surviving the neonatal period, and two were not born. The five indirect maternal deaths resulted in two stillborn babies, one early neonatal death and two survived the neonatal period.

The place of delivery, place of death and mode of transport to seek help is shown in Table 1. Forty-eight percent of all the babies that died were born at home, and $38 \%$ of the babies died at home. Among the 84 babies who died at or on the way to a health facility, 19 had been transported by a vehicle. The distribution of causes of death was fairly similar among deaths occurring at home and at health facilities $(P=0.16)$. Fifty-four percent of the infection-related cases and 36\% of the asphyxia related cases had been born at home, compared with $44 \%$ and $50 \%$ in a health facility $(P=0.3)$. Forty-four percent of the infection-related cases and 33\% of the asphyxia related cases died at home, compared with $46 \%$ and $53 \%$ at a health facility $(P=0.4)$.

We judged 34 (25\%) of the deaths to be possibly avoidable (Table 1). The proportion of avoidable deaths was higher among the late neonatal deaths (44\%) than among stillborn babies $(22 \%, P=0.03)$ and early neonatal deaths $(18 \%, P=0.01)$. Both the infection- and the asphyxia-related deaths were regarded as possibly avoidable in around a third of the cases (Table 1). Very few of the immaturity-related deaths and none of the congenital conditions were regarded as avoidable under the prevailing circumstances (Table 1). Among the 34 avoidable cases, 23 $(68 \%)$ were born at home and $22(65 \%)$ died at home (Table 1).

A patient-oriented factor was identified in $17(51 \%)$ of the 34 avoidable cases, 14 of them did not seek professional help in due time (Table 2). A provider-oriented avoidable factor was identified in $22(65 \%)$ of avoidable cases; in 12 of these cases, the woman should have been referred from the antenatal clinic to the hospital because of a disease or a risk factor (Table 2).

We identified at least one risk factor that could have been detected at routine antenatal visits in $84(62 \%)$ of the all the deaths (Table 1), with similar proportions in all the diagnostic categories. Among the 34 avoidable deaths, $26 \mathrm{had}$ at least one detectable antenatal risk factor. Only two of these women reported that a risk factor had been identified during the antenatal visits and only one remembered being referred to a higher level of care. For the others, the risk factors had either been overlooked at the clinic or the mother had not realised that she was referred for a risk factor to a higher level of care and therefore not reacted.

(C) RCOG 2003 Br J Obstet Gynaecol 110, pp. 616-623 
Table 1. Circumstances of $136^{\mathrm{a}}$ stillbirths and neonatal deaths in rural Tanzania, 1995-1996. The number of avoidable deaths is in parentheses.

\begin{tabular}{|c|c|c|c|c|}
\hline & \multirow[t]{2}{*}{ All (avoidable) } & \multicolumn{3}{|c|}{ Time of death } \\
\hline & & $\mathrm{SB}^{\mathrm{b}}$ (avoidable) & $\mathrm{END}^{\mathrm{b}}$ (avoidable) & LND $^{\mathrm{b}}$ (avoidable) \\
\hline \multicolumn{5}{|l|}{ Cause of death ${ }^{c}$} \\
\hline Infections & $53(19)$ & $25(5)$ & $11(3)$ & $17(11)$ \\
\hline Other causes/unclassifiable & $16(2)$ & $13(2)$ & $2(0)$ & $1(0)$ \\
\hline Congenital conditions & $9(0)$ & $4(0)$ & $4(0)$ & $1(0)$ \\
\hline External causes of death & $2(0)$ & $1(0)$ & $1(0)$ & 0 \\
\hline Sudden death & $1(0)$ & 0 & 0 & $1(0)$ \\
\hline Specific conditions & $1(1)$ & 0 & $1(1)$ & 0 \\
\hline No information & $2(2)$ & 0 & $1(1)$ & $1(1)$ \\
\hline Delivery roadside & $5(0)$ & $5(0)$ & 0 & 0 \\
\hline \multicolumn{5}{|l|}{ Place of death } \\
\hline Death at home & $52(22)$ & $21(7)$ & $17(5)$ & $14(10)$ \\
\hline Death at health facility & $75(10)$ & $34(6)$ & $30(4)$ & $11(0)$ \\
\hline Death roadside & $9(2)$ & $5(0)$ & $2(0)$ & $2(2)$ \\
\hline \multicolumn{5}{|l|}{ Transport to health facility } \\
\hline None & $55(11)$ & $24(5)$ & $20(4)$ & $11(2)$ \\
\hline Hospital ambulance & $14(0)$ & $6(0)$ & $6(0)$ & $2(0)$ \\
\hline Other vehicle & $5(0)$ & $2(0)$ & $3(0)$ & 0 \\
\hline Not applicable & $57(23)$ & $24(8)$ & $19(5)$ & $14(10)$ \\
\hline Says risk factor absent & $91(23)$ & $41(8)$ & $34(7)$ & $16(8)$ \\
\hline Did not attend $\mathrm{ANC}^{\mathrm{d}}$ & $3(2)$ & $1(0)$ & $1(1)$ & $1(1)$ \\
\hline No information & $34(7)$ & $15(4)$ & $11(1)$ & $8(2)$ \\
\hline \multicolumn{5}{|l|}{ Mother's perception of referral } \\
\hline Says she was referred & $17(1)$ & $6(0)$ & $8(0)$ & $3(1)$ \\
\hline Says she was not referred & $107(31)$ & $48(13)$ & $38(8)$ & $21(10)$ \\
\hline Did not attend $\mathrm{ANC}^{\mathrm{d}}$ & $3(2)$ & $1(0)$ & $1(1)$ & $1(1)$ \\
\hline No information & $9(0)$ & $5(0)$ & $2(0)$ & $2(0)$ \\
\hline
\end{tabular}

${ }^{a}$ Excluding three deaths with missing information on the variables in the table.

${ }^{\mathrm{b}} \mathrm{SB}=$ stillborn; END = early neonatal death; LND = late neonatal death.

${ }^{c}$ Deaths classified according to the International Collaborative Effort on Perinatal and Infant Mortality.

${ }^{\mathrm{d}}$ ANC $=$ antenatal care clinic.

\section{DISCUSSION}

In this review of perinatal and neonatal deaths, most of the cases were related to infections and asphyxia and almost $40 \%$ of the deaths occurred at home. Relatively few of the stillborn babies and the early neonatal deaths were avoidable under the prevailing circumstances, whereas among the late neonatal deaths, we found a higher proportion (41\%) to be avoidable. There was at least one risk factor that could have been detected antenatally in twothirds of the cases, but few of these mothers reported having been referred to a higher level of care.

Many studies on the causes of perinatal death are hospital based, which gives adequate medical and clinical information and also better potential for validation of verbal autopsies. However, those studies lack information on the

(C) RCOG 2003 Br J Obstet Gynaecol 110, pp. 616-623 
Table 2. Patient- and provider-oriented factors contributing to 34 avoidable stillbirths and neonatal deaths in rural Tanzania, 1995 - 1996.

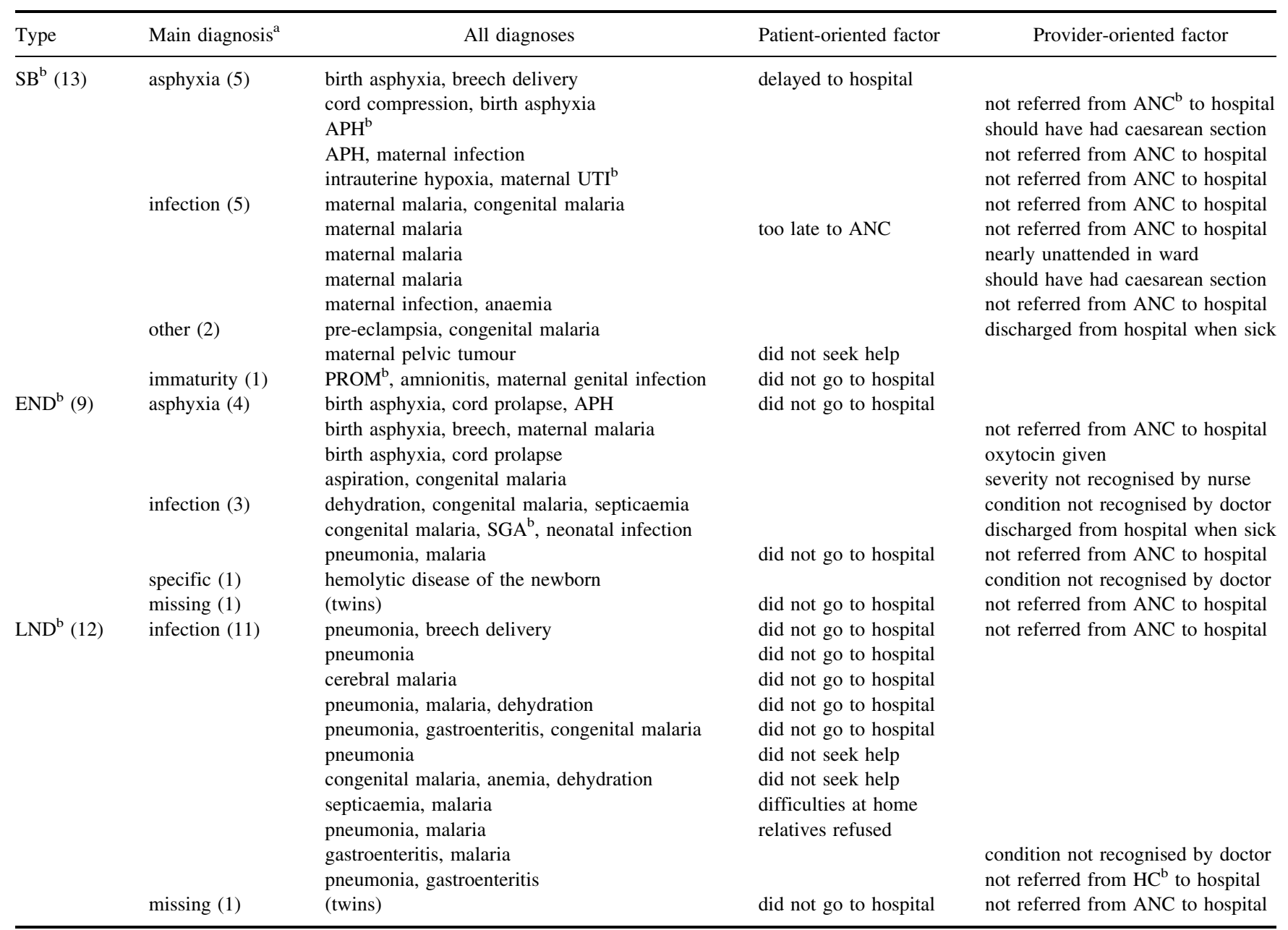

\footnotetext{
${ }^{a}$ Underlying cause of death grouped according to the International Collaborative Effort on Perinatal and Infant Mortality.

${ }^{\text {b }} \mathrm{SB}=$ stillbirth; END = early neonatal death; LND = late neonatal death; ANC = antenatal clinic; APH = antepartum haemorrhage; UTI = urinary tract infection; $\mathrm{PROM}=$ premature rupture of the membranes; $\mathrm{SGA}=$ small for gestational age; $\mathrm{HC}=$ health centre.
}

deaths occurring at home. The prospective population-based design in our study, with a low dropout rate, should ensure a fair representation of the perinatal problems in the community. The interviews were conducted by medical doctors familiar with the local epidemiology and well acquainted with the local culture. Nevertheless, our method of reaching a diagnosis leaves considerable room for inaccuracy, however meticulously the work is done ${ }^{20}$. Accurate observations and information are often inadequate to verify a diagnosis, although recall of mothers who have lost a child has been shown to be good even after a long time ${ }^{21}$. Furthermore, the signs and symptoms of diseases among neonates are often non-specific and similar for several different conditions. We could therefore only indicate tentative diagnoses. However, this weakness is counteracted by the use of broad categories of diagnoses ${ }^{21}$, which also has the advantage of focussing on the type of intervention needed to avoid death.

In many cases, several diagnoses contributed to the unfortunate outcome. For every death, we determined all likely diagnoses, but we used the most probable diagnosis as the underlying cause of death. This may somewhat distort the clinical picture, because it is often the combination of conditions that makes the baby unable to survive (e.g. a baby with low birthweight may also have asphyxia, and in the end, hypoglycaemia and hypothermia may cause the death). Hence, some of the known diagnoses that were assigned may have been given more or less importance than they deserve.

There were many deaths related to infections and asphyxia, which has also been found in other studies from sub-Saharan Africa ${ }^{22-24}$ including Tanzania ${ }^{10}$, and the contribution of congenital malformations was similar to what has been reported from other African countries. This contrasts the situation in many developed countries, where infections and asphyxia-related conditions are less common, while those more difficult to prevent or treat (i.e. congenital malformations and immaturity) are relatively more frequent ${ }^{25,26}$.

The proportion of possibly avoidable deaths observed in our study may seem small for a low-income country like 
Tanzania, and their prevention would only have reduced the perinatal mortality rate from 27 to $22 / 1000$ births. We judged 22 of the $136(16 \%)$ deaths to be, probably or possibly, due to factors in the health service provision. This is comparable with a health facility-based study in South Africa that indicated that $19 \%$ of the perinatal deaths were directly due to errors or omissions ('avoidable death') in the health service. The proportion was reduced after an intervention including the introduction of an audit process and the strengthening of the maternity service in the district, but there was no control group ${ }^{27}$. Other studies have identified 'avoidable factors' in $50-76 \%$ of perinatal deaths, but are not quite comparable because of the different definitions and methodology ${ }^{11,23,28}$. There may be some under-estimation of provider-oriented factors in our study, since information from the health facilities on the deaths was incomplete and a sound judgement on the standard of care cannot be based solely on verbal autopsies.

The low number of avoidable deaths must also be viewed in light of the relatively low perinatal mortality rate in the study area. Some 'avoidable' deaths may already have been prevented, bearing in mind the fairly well functioning antenatal, obstetric and neonatal services in the area, including an ambulance service with a timesaving VHFradio request system. Still, health care providers were responsible for more than half of the avoidable factors, both failure to refer the women from antenatal clinic to a higher level of care when needed and medical errors and omissions at hospital level. This indicates a potential for improving the antenatal routines and consultations, as well as obstetric routines in hospitals. Some cases that may have been treatable in principle, and therefore 'preventable' in a wider sense, were not defined as 'avoidable' in our study, because they would need changes in resource allocations and routines and systems. These may be interventions in pregnancy that have been shown to improve the outcome, like testing and treating syphilis ${ }^{29}$, using bed nets and presumptive treatment of malaria ${ }^{30}$.

The most common patient-oriented avoidable factor was failure to seek health services when the danger signs were recognised (Table 2). Many factors influence the decisionmaking process, and the need for professional health services is not the only issue to consider. Other issues are whether they have money, whether they have transport facilities, whether the husband agrees, whether the husband is around at all, whether the mother-in-law agrees, whether the local traditional doctor agrees, whether they have somebody to take care of the house and children and whether there is enough time to reach a hospital. Other factors that may influence their behaviour are previous bad experiences with health facilities and the staff, cultural barriers, peer pressure, fatalistic attitudes, poor roads and bad weather. In our study area, the woman's husband or mother-in-law was usually the decision-maker. Education of both women and men about reproductive health matters may influence the attitudes and practices around delivery.
New initiatives in antenatal care focus on delivery preparedness and complication readiness ${ }^{31}$, and male involvement should be an integral part of this strategy, since they are often decision-makers.

The most common cause of death was infections, with malaria predominating. Malaria (almost exclusively due to Plasmodium falciparum) is a common health problem in this area, and at the Haydom Lutheran Hospital, it was the most common cause of death among children as well as adults ${ }^{32}$. Previous international recommendations include weekly chloroquine prophylaxis in pregnancy, but recent revision recommends intermittent sulphadoxine-pyrimethamine ${ }^{30,33}$. In Kenya, intermittent treatment of presumptive malaria in pregnancy led to significantly increased birthweight. For mortality, the sample size was not sufficient to demonstrate a reduction in mortality, but the results were compatible with a protective efficacy of around $20 \%(95 \% \mathrm{CI}-17$ to 48) for perinatal mortality and around $40 \%$ for neonatal mortality (95\% CI -8 to 65$)^{34}$. Insecticide-treated bed nets have been shown to reduce mortality among children under five years of age $\mathrm{e}^{35}$, but they are rarely used in the study area. Their use should be encouraged in the antenatal care program.

Deaths due to septicaemia and pneumonia may be difficult to prevent. The symptoms may be vague and ambiguous even for experienced clinicians, and one cannot expect mothers to recognise the early danger signs. It is important that the threshold for contacting health facilities is low. Regular contact with health care personnel at antenatal clinics may reduce the threshold for professional contact. In areas with no hospitals, programs of integrated management of childhood infections have shown that even a home-based management of infections can reduce infant mortality ${ }^{36}$.

Some of the deaths related to asphyxia might have been prevented by professionally assisted delivery. Midwifeassisted home births could possibly prevent some of the deaths we have reviewed, but the policy of Tanzania does not include delivery services at home by midwives. The effect of training traditional birth attendants on the outcome of pregnancy has been rather disappointing ${ }^{37}$ and may sometimes even be harmful by adopting practices meant for hospital settings ${ }^{38}$, but may play a role in closer collaboration with midwives and doctors ${ }^{39}$.

Congenital malformations were found in $6 \%$ of cases, including some neural crest anomalies. In our study area, a relatively high proportion (14-33\%) of pregnant women had low serum folate ${ }^{40}$. It has been shown that periconceptional folic acid supplementation can prevent some neural tube defects ${ }^{41}$, but it may be difficult in this area as women seldom 'prepare' for pregnancy and usually pay the first visit to the health system or antenatal clinics during the second trimester ${ }^{42}$. General dietary advice may be theoretical because of seasonal shortages of various vegetables.

There was a quite high proportion of deaths in this series of pregnant women. Their death rate was much higher than 
the maternal mortality ratio in the area. Many of the problems that affect the newborn may also harm the mother (e.g. malaria, antepartum bleedings, eclampsia). A mother who loses her baby should be observed carefully.

Antenatal care has previously focussed on screening for high risk pregnancies in order to give those in need a follow up schedule at a higher level of health care, but the strategy has shortcomings ${ }^{43}$. We have shown that most of the deaths $(62 \%)$ had a risk factor identifiable at the antenatal clinic, but more than a third did not have any risk factor and would not have been identified through ordinary antenatal care. It was even more discouraging that most of the women with risk factors were not made aware of it. Similar findings have been presented in other studies in Tanzania ${ }^{44}$ and emphasises the importance of a good dialogue between staff and pregnant women at antenatal clinics, making sure that messages are understood.

\section{CONCLUSION}

This study highlights the importance of a holistic approach to ensure a satisfactory outcome of pregnancy for women in resource limited settings. The general public needs to be educated to understand that obstetric complications are emergencies and that every pregnancy is 'at risk'. The antenatal clinics must continue to play an important role in screening and referral of the women at risk or with complications to a higher level of care. Improved dialogue between antenatal clinic staff and the pregnant women should aim to make messages understood. Antenatal care should focus on preparing the women for delivery and educating them and their husbands to be ready for unexpected complications. Finally, effective antenatal care presupposes a functioning referral level with emergency obstetric care, which needs to be credible, affordable and rapidly available.

\section{Acknowledgements}

The study was funded by the Norwegian Research Council and the Centre for International Health at the University of Bergen, Norway. The authors thank the Regional Development Director (RDD), Regional Medical Officer and Regional Maternal and Child Health coordinator in Arusha Region, the District Commissioners, District Medical Officers, Maternal and Child Health coordinators and staff at all the health centres and dispensaries and at the local division, the ward and village administrators in Hanang and Mbulu districts for their assistance. In addition, the authors thank the Mbulu Diocese of the Evangelical Lutheran Church of Tanzania for their support. Many ten-cell leaders and citizens gave invaluable assistance and commitment tracing the bereaved households. The authors are indebted to the project staff, the administration at
Haydom Lutheran Hospital, the staff of the hospital and the staff at the garage for their generous assistance. The authors also thank Dr Isack Malleyeck, Dr Emmanuel Mayega and Herman Malleyeck for their help with the medical records. Most of all, the authors thank the participants of the study for their willingness to share their knowledge with us.

\section{References}

1. World Health Organization. World Health Report 2001. Geneva: WHO, 2001.

2. Tanner M, de Savigny D, Mayombana C, et al. Chapter 19: Morbidity and mortality at Kilombero, Tanzania, 1982-88. In: Feachem RG, Jamison DT, editors. Disease and Mortality in Sub-Saharan Africa. Washington, DC: World Bank, 1991:286-305.

3. Möller B, Lushino O, Kabukoba J, et al. A prospective area-based study of the outcome of pregnancy in rural Tanzania. Ups J Med Sci 1989;94:101-109.

4. Walraven GE, Mkanje RJ, van Roosmalen J, van Dongen PW, Dolmans WM. Comparison of perinatal outcome in rural Tanzania as obtained from a prospective community-based survey and hospital data. Trop Geogr Med 1994;46:11-13.

5. Kilonzo A, Kouletio M, Whitehead SJ, Curtis KM, McCarthy BJ. Improving surveillance for maternal and perinatal health in 2 districts of rural Tanzania. Am J Public Health 2001;91:1636-1640.

6. Baird D. Perinatal mortality. Lancet 1969;1:511-515.

7. Loudon I. On maternal and infant mortality, 1900-1960. Soc Hist Med 1991;4:29-73.

8. Andersson T, Högberg U, Bergström S. Community-based prevention of perinatal deaths: lessons from nineteenth-century Sweden. Int $J$ Epidemiol 2000;29:542-548.

9. Loudon I. Maternal mortality in the past and its relevance to developing countries today. Am J Clin Nutr 2000;72:241s-246s.

10. van Roosmalen J. Perinatal mortality in rural Tanzania. Br J Obstet Gynaecol 1989;96:827-834.

11. Ward HR, Howarth GR, Jennings OJ, Pattinson RC. Audit incorporating avoidability and appropriate intervention can significantly decrease perinatal mortality. S Afr Med J 1995;85:147-150.

12. World Health Organization. Coverage of Maternity Care. Geneva: WHO, 1997.

13. Olsen BE, Hinderaker SG, Lie RT, Bergsjø P, Gasheka P, Kvåle G. Maternal mortality in northern rural Tanzania: assessing the completeness of various information sources. Acta Obstet Gynecol Scand 2002;81:301-307.

14. Hinderaker SG, Kruger C, Olsen BE, Naman N, Bergsjø P, Olsen OH. Low HIV-seroprevalence in pregnant women in a rural area in Tanzania. Acta Obstet Gynecol Scand 2001;80:1152-1153.

15. Smith PG, Morrow RH. Methods for Field Trials of Interventions Against Tropical Diseases. New York: Oxford University Press, 1991.

16. Wigglesworth JS. Monitoring perinatal mortality. A pathophysiological approach. Lancet 1980;2:684-686.

17. Cole S, Hartford RB, Bergsjø P, McCarthy B. International collaborative effort (ICE) on birth weight, plurality, perinatal, and infant mortality. III: A method of grouping underlying causes of infant death to aid international comparisons. Acta Obstet Gynecol Scand 1989; 68: $113-117$.

18. Dean AG, Dean JA, Coulombier D, et al. Epi Info, Version 6: A Word Processing, Database, and Statistics Program for Epidemiology on Microcomputers. Atlanta, Georgia: Centers for Disease Control and Prevention, 1994.

19. SPSS. SPSS Base 9.0: User's Guide. Chicago, Illinois: SPSS, 1999.

20. Snow RW, Armstrong JR, Forster D, et al. Childhood deaths in Africa: uses and limitations of verbal autopsies. Lancet 1992;340:351-355.

21. Chandramohan D, Maude GH, Rodrigues LC, Hayes RJ. Verbal 
autopsies for adult deaths: issues in their development and validation. Int J Epidemiol 1994;23:213-222.

22. Nordbeck HJ, Voorhoeve AM, van Ginneken JK. Chapter 19: The use of perinatal mortality data in antenatal screening. In: van Ginneken JK, Muller AS, editors. Maternal and Child Health in Rural Kenya. Nairobi: African Medical Research Foundation, 1984:241-255.

23. Pattinson RC, Makin JD, Shaw A, Delport SD. The value of incorporating avoidable factors into perinatal audits. S Afr Med J 1995;85: $145-147$.

24. Slutsker L, Bloland P, Steketee RW, Wirima JJ, Heymann DL, Breman JG. Infant and second-year mortality in rural Malawi: causes and descriptive epidemiology. Am J Trop Med Hyg 1996;55:77-81.

25. Proceedings of the International Symposium on Perinatal and Infant Mortality. Bethesda, Maryland, 1990.

26. Irgens L. Births in Norway through 30 years. Bergen: Medical Birth Registry of Norway, 1997.

27. Wilkinson D. Reducing perinatal mortality in developing countries. Health Policy Plan 1997;12:161-165.

28. De Muylder X. Perinatal mortality audit in a Zimbabwean district. Paediatr Perinat Epidemiol 1989;3:284-293.

29. Rotchford K, Lombard C, Zuma K, Wilkinson D. Impact on perinatal mortality of missed opportunities to treat maternal syphilis in rural South Africa: baseline results from a clinic randomized controlled trial. Trop Med Int Health 2000;5:800-804.

30. Garner P, Gulmezoglu AM. Prevention versus treatment for malaria in pregnant women. Cochrane Database Syst Rev 2000;2.

31. Kinzie B, Stanton C. Antenatal care: old myths, new realities. Slide presentation, USAID/Washington Maternal Health (PHN/SO2) Technical Series. Available at: http://www.mnh.jhpiego.org/updates/2001/ mnhoct01.htm (4 February 2002).

32. Haydom Lutheran Hospital. Annual Report 1996. Mbulu: Haydom Lutheran Hospital, 1997.

33. United Republic of Tanzania Ministry of Health. National Guidelines for Malaria Diagnosis and Treatment. Dar es Salaam: Ministry of Health, 2000.
34. Shulman CE, Dorman EK, Cutts F, et al. Intermittent sulphadoxinepyrimethamine to prevent severe anaemia secondary to malaria in pregnancy: a randomised placebo-controlled trial. Lancet 1999;353: 632-636.

35. Lengeler C. Insecticide-treated bednets and curtains for preventing malaria. Cochrane Database Syst Rev 2000;2.

36. Bang AT, Bang RA, Baitule SB, Reddy MH, Deshmukh MD. Effect of home-based neonatal care and management of sepsis on neonatal mortality: field trial in rural India. Lancet 1999;354:1955-1961.

37. Greenwood AM, Bradley AK, Byass P, et al. Evaluation of a primary health care programme in The Gambia. I. The impact of trained traditional birth attendants on the outcome of pregnancy. J Trop Med Hyg 1990;93:58-66.

38. Jahn A, Carvalho I, Kalinga MJ. Evaluating traditional midwife training programs: lessons learned from Tanzania. Int J Gynaecol Obstet 2001;73:277-278.

39. Kamal IT. The traditional birth attendant: a reality and a challenge. Int J Gynecol Obstet 1998;63(Suppl 1):S43-S52.

40. Hinderaker SG, Olsen BE, Lie RT, et al. Anemia in pregnancy in rural Tanzania: associations with micronutrients status and infections. Eur J Clin Nutr 2002;56:192-199.

41. Lumley J, Watson L, Watson M, Bower C. Periconceptional supplementation with folate and/or multivitamins for preventing neural tube defects [Cochrane review]. Cochrane Database Syst Rev 2001;3.

42. Hinderaker SG, Olsen BE, Bergsjø P, Lie RT, Gasheka P, Kvåle G. Anemia in pregnancy in the highlands of Tanzania. Acta Obstet Gynecol Scand 2001;80:18-26.

43. Carroli G, Rooney C, Villar J. How effective is antenatal care in preventing maternal mortality and serious morbidity? An overview of the evidence. Paediatr Perinat Epidemiol 2001;15:1-42.

44. Jahn A, Kowalewski M, Kimatta SS. Obstetric care in southern Tanzania: does it reach those in need? Trop Med Int Health 1998;3: 926-932.

Accepted 21 January 2003 